\title{
Do pathogenic bacteria encode more secreted proteins than their non-pathogenic relatives?
}

\author{
Ahmed Abo-Bakr Mahmoud', Ramy Karam Aziz ${ }^{2^{*}}$ \\ From UT-ORNL-KBRIN Bioinformatics Summit 2010 \\ Cadiz, KY, USA. 19-21 March 2010
}

\section{Background}

Pathogenic and non-pathogenic bacteria secrete proteins for nutrient acquisition, cell-cell communication, and niche adaptation [1]. We hypothesized that pathogenic bacteria may encode larger fractions of secreted proteins (fsp) than their non-pathogenic relatives, assuming that pathogens might be under selective pressure to secrete virulence proteins involved in host immune evasion, invasion, and toxigenesis. To test this hypothesis, we compared the Sec-dependent fsp of various gram-positive and gram-negative bacteria and investigated the relation between the fsp and pathogenic potential of an organism.

\section{Methods}

We developed a pipeline that starts by a Perl script that truncates protein sequences to 70 amino acids or fewer followed by the application of existing signal prediction tools [2-4] and ends by the statistical analysis of the prediction data. For subsequent comparative secretome analyses, we used both the hidden Markov models- and the neural networks-based methods implemented in the SignalP 3.0 algorithm [2] (URL: http://www.cbs.dtu.dk/ services/SignalP) with modified thresholds. We used DataDesk (Data Description, Inc., Ithaca, NY; URL: http://www.datadesk.com) for all statistical analyses (including correlation analysis, analysis of variance, and multivariate analysis) and for plotting the results.

\section{Results}

We determined the theoretical secretomes of 176 chromosomes and 115 plasmids in five gram-positive and five gram-negative bacterial genera containing

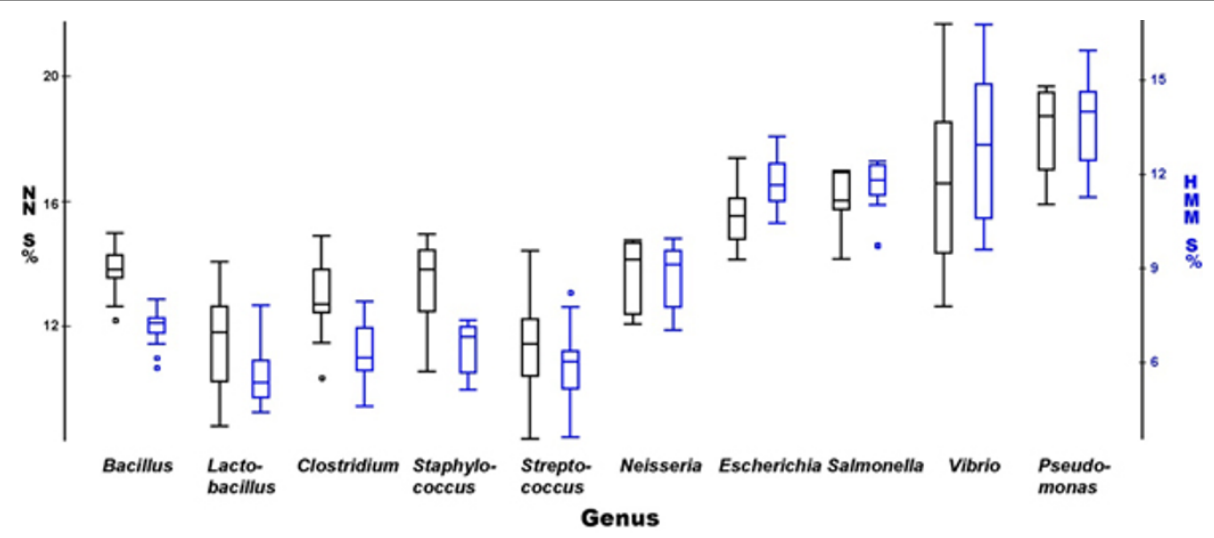

Figure 1 Predicted fractions of secreted proteins by the chromosomes of ten bacterial genera with pathogenic and non-pathogenic member. $\mathrm{NN}=$ neural networks method; $\mathrm{HMM}=$ hidden Markov models method.

* Correspondence: ramy.aziz@salmonella.org

2Department of Microbiology and Immunology, Faculty of Pharmacy, Cairo

University, Cairo 11562, Egypt 


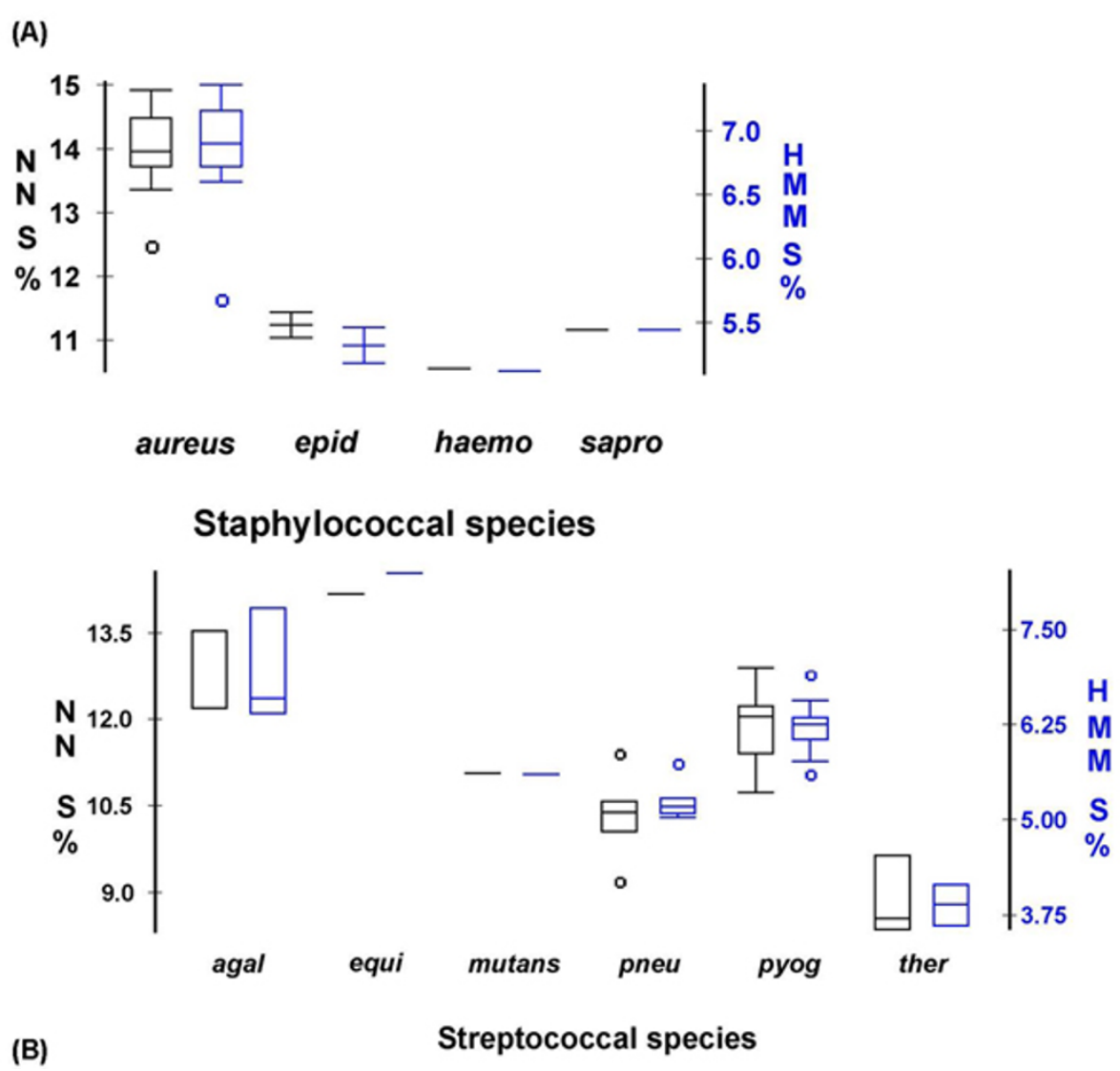

Figure 2 Pathogenic Gram-positive cocci encode larger fractions of secreted proteins than non-pathogenic relatives. NN = neural networks method; HMM = hidden Markov models method. (A) Staphylococcal species (epid = epiderimidis; haemo = haemolyticus; sapro = saprophyticus). (B) Streptococcal species (aga = agalactiae; pneu = pneumoniae; pyog = pyogenes; ther = thermophilus) $P$ values: aureus vs. all: $P<10-6$; ther vs. all: $P=0.002$ (NN), 0.0001 (HMM); agal vs. $p y o g: P=0.012(\mathrm{NN}), 0.003(\mathrm{HMM})$.

pathogenic and non-pathogenic members (Figure 1). Our analysis showed significant differences in chromosomally encoded fsp between gram-positive and gramnegative bacteria (chromosomes of gram-negative bacteria have larger fsp), while there was no particular pattern in plasmid-encoded fsp. Whereas the overall difference between pathogenic and non-pathogenic species was not statistically significant, significant correlation was observed between fsp and pathogenesis in gram-positive cocci. For example, pathogenic Staphylococcus aureus have higher fsp than other staphylococci, while the non-pathogenic Streptococcus thermophilus has the lowest fsp of all streptococci (Figure 2).

\section{Conclusion}

We developed a pipeline for the determination and comparison of fractions of secreted proteins in bacterial genomes, and observed significant differences between pathogenic and non-pathogenic species of staphylococci and streptococci.
Author details

${ }^{1}$ Maternity Governmental Hospital, Kuwait City, 85860, Kuwait. ²Department of Microbiology and Immunology, Faculty of Pharmacy, Cairo University, Cairo 11562, Egypt.

Published: 23 July 2010

\section{References}

1. Gennity JM, Inouye M: Protein secretion in bacteria. Curr Opin Biotechnol 1991, 2:661-667.

2. Bendtsen JD, Nielsen $H$, von Heijne $G$, Brunak S: Improved prediction of signal peptides: SignalP 3.0. J Mol Biol 2004, 340:783-795.

3. Emanuelsson O, Brunak S, von Heijne G, Nielsen H: Locating proteins in the cell using TargetP, SignalP and related tools. Nat Protoc 2007, 2:953-971.

4. Zhou M, Boekhorst J, Francke C, Siezen RJ: LocateP: genome-scale subcellular- location predictor for bacterial proteins. BMC Bioinformatics 2008, 9:173.

doi:10.1186/1471-2105-11-S4-P28

Cite this article as: Mahmoud and Aziz: Do pathogenic bacteria encode more secreted proteins than their non-pathogenic relatives?. BMC Bioinformatics 2010 11(Suppl 4):P28. 